\title{
Author Correction: HuR regulates telomerase activity through TERC methylation
}

Hao Tang ${ }^{1,2}$, Hu Wang ${ }^{3,4}$, Xiaolei Cheng ${ }^{1}$, Xiuqin Fan'1, Fan Yang ${ }^{3}$, Mengmeng Zhang ${ }^{5}$, Yanlian Chen ${ }^{6}$, Yuyang Tian ${ }^{6}$, Cihang Liu', Dongxing Shao', Bin Jiang ${ }^{1}$, Yali Dou7, Yusheng Cong ${ }^{3,4}$, Junyue Xing ${ }^{1}$, Xiaotian Zhang ${ }^{1}$, Xia Yi ${ }^{1}$, Zhou Songyang ${ }^{6}$, Wenbin Ma (i) ${ }^{6}$, Yong Zhao ${ }^{6}$, Xian Wang ${ }^{2}$, Jinbiao Ma (D) ${ }^{5}$, Myriam Gorospe $^{8}$, Zhenyu Ju ${ }^{3,4} \&$ Wengong Wang (1) ${ }^{1}$

Correction to: Nature Communications; https://doi.org/10.1038/s41467-018-04617-7; published online 07 June 2018

In the original version of this Article, the affiliation details for Fan Yang incorrectly included 'Leibniz Institute for Age Research - Fritz Lipmann Institute, Friedrich-Schiller University of Jena, Jena, 07745, Germany'. This has now been corrected in both the PDF and HTML versions of the Article.

Published online: 10 July 2018

Open Access This article is licensed under a Creative Commons Attribution 4.0 International License, which permits use, sharing, adaptation, distribution and reproduction in any medium or format, as long as you give appropriate credit to the original author(s) and the source, provide a link to the Creative Commons license, and indicate if changes were made. The images or other third party material in this article are included in the article's Creative Commons license, unless indicated otherwise in a credit line to the material. If material is not included in the article's Creative Commons license and your intended use is not permitted by statutory regulation or exceeds the permitted use, you will need to obtain permission directly from the copyright holder. To view a copy of this license, visit http://creativecommons.org/licenses/by/4.0/.

(C) The Author(s) 2018

\footnotetext{
${ }^{1}$ Department of Biochemistry and Molecular Biology, Beijing Key Laboratory of Protein Posttranslational Modifications and Cell Function, School of Basic Medical Sciences, Peking University Health Science Center, 38 Xueyuan Road, Beijing 100191, China. ${ }^{2}$ Department of Physiology and Pathophysiology, School of Basic Medical Sciences, Peking University Health Science Center, 38 Xueyuan Road, Beijing 100191, China. ${ }^{3}$ Key Laboratory of Regenerative Medicine of Ministry of Education, Institute of Aging and Regenerative Medicine, Jinan University, Guangzhou 510632, China. ${ }^{4}$ Institute of Aging Research, Hangzhou Normal University, School of Medicine, Hangzhou 311121, China. ${ }^{5}$ Department of Biochemistry, School of life Sciences, Fudan University, 2005 Road Songhu, Shanghai 200433, China. ${ }^{6}$ Key Laboratory of Gene Engineering of the Ministry of Education, State Key Laboratory of Biocontrol, School of Life Sciences, Sun Yat-sen University, Guangzhou 510006, China. ${ }^{7}$ Department of Pathology and Biological Chemistry, University of Michigan, 1301 Catherine Street, Ann Arbor, MI 48105, USA. ${ }^{8}$ Laboratory of Genetics and Genomics, National Institute on Aging, National Institutes of Health, 251 Bayview Blvd., Baltimore, MD 21224, USA. Correspondence and requests for materials should be addressed to Z.J. (email: zhenyuju@163.com) or to W.W. (email: wwg@bjmu.edu.cn)
} 